Subject strapline: Viral pathogenesis

\title{
Viral programming of progenitor cell commitment
}

\author{
Author 1
}

1 Matthew B. Reeves

\section{Standfirst}

Human cytomegalovirus infects pluripotent haematopoetic progenitor cells, yet it is latent in cells of the monocyte/myeloid lineage. A new study reveals that HCMV achieves this by actively re-programming the infected progenitor cell into a unique monocyte subset, enabling the successful lifelong persistence of HCMV in its host.

\section{Main text}

Viruses exist in a hostile world. Their hosts are armed with multiple defence mechanisms that limit viral replication, requiring viruses to employ numerous countermeasures in order to survive. The life style choices of herpes viruses, such as human cytomegalovirus (HCMV), exacerbate this problem: through latency, they establish a lifelong association with their host, leaving them constantly under threat from immune surveillance. One potential solution for any pathogen under these circumstances is to hide: indeed, for many years, it was considered that the establishment of herpes virus latency relied on what was perceived to be the 'quiescent' nature of the infection. However, recent studies have revealed that latent HCMV is far from silent and the virus in fact relies upon the maintenance of a very distinct transcriptional profile in order to maintain its latent state. In this issue of Nature Microbiology Zhu and colleagues describe how one of the gene products expressed during HCMV latency, US28, manipulates host cell identity and immune function to promote persistence of $\mathrm{HCMV}^{1}$.

In order to be transmitted to a new host, herpes viruses must periodically reactivate from their latent infection. HCMV was thought to doso by 'piggybacking' onto the normal differentiation pathways that generate the cells of the myeloid lineage. Thus, HCMV would establish latency in haematopoietic progenitor cells and only reactivate to produce infectious progeny when these cells differentiate into mature myeloid cells. Two immediate questions arose from these observations: why, despite the capacity of the infected progenitor to generate all blood cell types, is the virus segregated with the myeloid lineage? What are the biological consequences 
of persisting and reactivating in a cell type that is the epicentre of the immune response and might thus be assumed to limit viral replication? Zhu et al now demonstrate that the virally encoded gene product US28 re-programms haematopoietic progenitor cells into a specialised myeloid monocyte subset with unique immune-modulatory properties, providing an answer to the first question and a strategy to evade the inherent problems associated with the second.

US28 is a virally encoded G-protein-coupled receptor that has been demonstrated to play an important role in cell signalling and migration, as well as being an important mediator of HCMVinduced vascular pathology 2 . The expression of US28 during latent HCMV infection was reported in $2001^{3}$, but remained largely unexplored until a slew of recent studies revealed a key role for this protein in the establishment of latency and the regulation of reactivation in multiple in vitro models ${ }^{4-6}$. The effects of US28 were linked with its capacity to differentially modulate cell signalling pathways in a cell-type and ligand-specific manner. A tantalising example of this phenomenon was the differential regulation of signalling pathways implicated in HCMV reactivation: US28 inhibits their activation in latent cells but supports the activation of the very same pathways in reactivating cells ${ }^{6}$.

In the current study, Zhu et al confirm the key role of US28 signalling in the establishment of latency. Specifically, they demonstrate that US28-dependent activation of STAT3 signalling and the concomitant upregulation of nitric oxide (NO) production in the infected cells promotes latency, and that loss of this STAT-3/NO axis from the cell is sufficient to prevent the establishment of latency using in vitro models. Furthermore, they demonstrate that normal cellular differentiation - an established inducer of HCMV reactivation - coincides with a downregulation of NO levels in the differentiating cells. The precise mechanism by which NO maintains latency remains unclear. Importantly, however, NO is a known inhibitor of T cell proliferation and Zhu et al show that its induction is required for the immune suppressive phenotype of the virally infected cells,, potentially protecting the latently infected cell from the immune response.

The segregation of viral genomes into cells of the myeloid lineage is a hallmark of HCMV latency, yet the underlying mechanism is not really understood. Zhu et al argue that this myeloid monocyte commitment of infected progenitor cells is not a chance event but instead is programmed by the latent virus and US28. Perhaps the most intriguing aspect of the study, however, is the identity of the latently infected monocyte that was generated from the infected progenitor cells. Monocytes are typically short lived; however, the findings of Zhu et al suggest that HCMV directs the differentiation of progenitor cells towards an immune-suppressive monocyte phenotype with substantially increased longevity in vitro. Conducting animal experiments with HCMV in vivo is incredibly difficult due to its highly restricted host range; however, the demonstration that HCMV genomes were substantially enriched in this rare monocyte subset in naturally infected individuals provided powerful support for their in vitro observations. Indeed, studies of natural latency are hindered by the relative scarcity of latently infected cells present in blood samples. Thus, the discovery of a potential method to enrich for naturally latently infected cells could revolutionise the field and the type of questions that we can now ask about the virus in its natural state in vivo. 
Of course, the devil's advocate may argue that enrichment in this lineage does not preclude the importance of other lineages. For example, if the turnover rates of two cell types both carrying the viral genome are markedly different, then you could expect an accumulation of latently infected cells in any compartment that turns over more slowly and is therefore never being depleted. Additionally, the immune-suppressive capacity of these cells may contribute to the persistence of the virus in them - the virus will predominate in a compartment that is better equipped to evade immune surveillance, but a transient residence in other cell types still may play an important role in the biology of the virus in vivo.

Despite these caveats, the potential identification of a predominant cellular site of latency in vivo that is dependent on US28 activity for its formation introduces the possibility of eliminating these cells in individuals for whom reactivation may be dangerous, such as transplant recipients. Indeed, a proof of concept study has shown that latently expressed US28 can be targeted using an immuno-toxin based approach to eliminate infected cells in vitro ${ }^{6}$.

Perhaps a more academic question is how other bone marrow-derived cell types fit into this model of HCMV-driven differentiation to an immune-suppressive monocyte phenotype? Dendritic cells (DCs) clearly support HCMV reactivation in vitro and ex vivo ${ }^{7}$. However, it is hypothesised that DCs originate from a distinct precursor in the bone marrow rather than via a monocyte intermediate during normal haematopoiesis. Perhaps this argues for the concomitant infection of DC committed progenitors alongside more primitive haematopoietic cells? That is, perhaps US28 can only programme the immune-suppressive monocyte phenotype in progenitors yet to be committed to a certain lineage? Whilst this hypothesis would explain the detection of HCMV in monocyte and DC compartments in peripheral blood, it does not explain the known absence of HCMV in the lymphoid compartment. Thus a further possibility is that a viral gene function is active in the DC progenitors that support their survival and differentiation, whereas lymphocyte progenitors do not support latent gene expression at all and thus the virus is eliminated from these cells. We could also be too rigid in the classification we give to different cell types. Perhaps the virus, through its ability to manipulate progenitor cells, will reveal novel insights into the ontogeny and plasticity of the haematopoietic system, as well as the biology of differentiated myeloid cells.

\section{Competing Interests}

The author declares no competing financial interests

\section{Figure legend}


Figure 1. Human cytomegalovirus and haematopoiesis. Haematopoiesis underpins the genesis of all mature blood cell types from the haematopoietic progenitor cell (HPC) niche resident in the bone marrow. The precise cell type that HCMV infects in the haematopoietic cell lineage is unknown, but the virus has been found in the monocyte and DC compartments in peripheral blood. HCMV may infect the HPC directly (as implicated by Zhu and colleagues), infect committed monocyte and DC precursors, or infect all cells in the bone marrow. The expression of US28 promotes the formation of immune suppressive monocytes in a progenitor cell subset via STAT-3 activation. Common Myeloid Progenitor (CMP), Common Lymphoid Progenitor (CLP), Nitric Oxide (NO), Interleukin-10 (IL-10), Dendritic Cell (DC)

\section{References}

1. Zhu et al. Nature Microbiology http:// (2018)

2. Boomker J.M., van Luyn M.J., The T.H., de Leij L.F. \& Harmsen M.C. Rev. Med. Virol. 15(4):269-82 (2005)

3. Beisser P.S., Laurent L., Virelizier J.L. \& Michelson S. J. Virol. 75(13):5949-57 (2001)

4. Humby M.S. \& O’Connor C.M. J. Virol. 90(6):2959-70 (2015)

5. Krishna B.A. et al Nat. Commun. 8:14321. doi: 10.1038/ncomms14321. (2017)

6. Krishna B.A. et al mBio 8(6). pii: e01754-17. doi: 10.1128/mBio.01754-17. (2017)

7. Reeves M.B. \& Sinclair J.S. J Virol. 87(19):10660-7 (2013) 